\title{
ICU Management of a patient with Anaphylaxis
}

\author{
Kudavidanage BP \\ Department of Anaesthesia - Teaching Hospital Anuradhapura
}

\begin{abstract}
Hypersensitivity reactions might occur during anaesthesia. Muscle relaxants have the highest frequency of inducing anaphylaxis. Rocuronium is the most common out of all muscle relaxants. We experienced a case of anaphylaxis occurring due to rocuronium during anaesthesia in a patient who is coming for a nephrectomy at Queens Hospital, London, UK. We managed the episode of anaphylaxis according to guidelines established by Association of Anaesthetists of Great Britain and Ireland.
\end{abstract}

\section{Introduction}

Hypersensitivity reactions that might occur during anaesthesia, i.e., anaphylaxis and anaphylactoid reaction have been reported to have a variable degree of incidence. But it has been reported to be approximately $1 / 3,000$ 20,000 [1] and its mortality has been reported to be $3-6 \%$ [1]. Of the drugs which are used for anaesthesia, muscle relaxants have the highest frequency of inducing anaphylaxis. In addition, substances such as latex or antibiotics can also induce the occurrence of anaphylaxis.

On the other hand, the occurrence of anaphylaxis due to anaesthetic agents has been frequently reported in men in their fifties and women in their forties. In paediatric patients aged ten years or younger, it occurs to such a rare extent as $<4 \%$ of total cases of anaphylaxis [3]. In the mid-1990s, rocuronium bromide (one of the non depolarizing aminosteroid muscle relaxants with a short action time) has been introduced in a clinical setting. As described here, according to the increased use of rocuronium, although not yet available in Sri Lanka, the occurrence of Rocuronium anaphylaxis has been reported to rise [1].
But most of these cases have been reported to occur in middle-aged and elderly people. We experienced a case of anaphylaxis occurring due to rocuronium during anaesthesia in a patient who is coming for a nephrectomy.

\section{Case History \\ Preoperative assessment}

Our patient was a 69 year old female Caucasian coming for an elective radical laparoscopic nephrectomy for multi locular renal cell carcinoma at Queens Hospital, London. She gave a history of well controlled hypertension without any end organ damage. She did not have any other medical problems. She had good exercise tolerance. She was on regular antihypertensives, namely Ramipril $2.5 \mathrm{mg}$ once daily, Bendroflumethiazole $2.5 \mathrm{mg}$ once daily and anti depression Citalopram 20mg nocte.

She had undergone general anaesthesia for repair of detached retina in 1990. She received Thiopentone, Atracurium and Fentanyl for general anaesthesia and there were no reported complications. She didn't give any history of allergy to any of the medications or food.

On examination she was an averagely built lady. She was not pale. On assessment of her air way, she didn't present with a difficult airway. On cardiovascular assessment pulse rate was 65 beats per minute and blood Pressure was 170/85. Heart was in dual rhythm and no murmurs detected. Respiratory and central nervous systems were normal.

Blood investigations were all normal except for haemoglobin which is 12.5 . Consent was taken for surgery after explaining the risks of general anaesthesia and laparoscopic nephrectomy. 


\section{Anaesthetic procedure}

After establishing 16G intravenous access on the dorsum of the right hand and stating $0.9 \%$ intravenous infusion, routine monitoring was established. Initial pulse rate was $70 \mathrm{bpm}$, blood pressure $180 / 90 \mathrm{mmHg}$ and saturation was $96 \%$ on induction. Patient was induced intravenously using Propofol 130mg, Fentanyl $100 \mu \mathrm{g}$ and muscle relaxant was induced with Rocuronium 40 mg intravenously. Patient was intubated with size 8 end tracheal tube and tube was secured after checking the position. As soon as the patient was induced, saturation in the pulse oxymeter dropped to $60 \%$ and air way pressure increased to $40 \mathrm{cmH}_{2} \mathrm{O}$. Blood pressure also dropped to 60/30 and severe bradycardia was noticed. Patient was clinically flushed on examination.

The initial differential diagnosis was a drop in blood pressure due to overdose of induction agents and anaphylactic reaction to induction agents or muscle relaxants.

Intravenous fluids especially $500 \mathrm{ml}$ of colloids were transfused as a bolus, followed by injection of ephedrine and Metaraminol boluses to improve blood pressure. Epheneprene boluses were required to maintain blood pressure as blood pressure was low in spite of previous treatment. This was followed by Epheneprine infusion which was titrated according to the response to maintain the blood pressure.

Management of the patient was done by following the guidelines for anaphylaxis management by the Association of Anaesthetists in Great Britain and Ireland. Accordingly Hydrocortisone 100mg and Ranitidine 50mg was given and Chlopheniramine $10 \mathrm{mg}$ also given intravenously.

\section{Post op management}

Patent was admitted to intensive care for further management with intermittent positive pressure ventilation and infusions of Ephenephrene, Remifentanyl and Midazolam. On admission she was on $50 \%$ oxygen maintaining $100 \%$ saturation with $\mathrm{PaO}_{2}$ of 54 $\mathrm{kPa}$ and $\mathrm{PaCO}_{2}$ of $7.15 \mathrm{kPa}$. Chest $\mathrm{X}$ ray was clear without any evidence of pulmonary oedema. She was tachycardic with blood pressure maintained by adrenalin infusion. She was on Midazolam and Remifentanyl infusion for sedation with pupils reacting equally to light. Initial investigations were within normal range except for the serum potassium which was 3.2 $\mathrm{mmol} / \mathrm{l}$, glucose of $8.4 \mathrm{mmol} / \mathrm{l}$ with normal clotting profile. In addition serum tryptase was taken immediately after admission to the ICU, 6 hours after and 24 hours after the admission to ICU. Routine observation was carried out in the ICU with close monitoring for further evidence of anaphylaxis.

Once haemodynamically stable she was gradually weaned off from adrenaline infusion and ventilatory support was reduced. Potassium was replaced intravenously. Advice for further management was taken from the regional allergic centre at the allergic clinic at the Guy's Hospital in London.

She was referred to the allergic clinic at Guy's Hospital for further investigations, where she underwent skin testing and RAST testing. Skin testing was strongly positive for Rocuronium and Suxamethonium, while mildly positive for Vecuronium, Mivacurium and borderline positive for Atracurium. Investigations established that Cisatracurium, Pancuronium and Propofol are negative for the reaction and were safe to use. Therefore it was confirmed that the anaphylactic reaction was due to the Rocuronium which was used at the induction.

She subsequently successfully underwent the surgery with Propofol, Pancuronium and fentanyl. 


\section{Discussion}

We experienced a case in which anaphylaxis was strongly suspected based on hypotension, tachycardia and hypoxia due to the use of Rocuronium which was used for general anaesthesia.

Two types of immediate hypersensitivity reactions including anaphylaxis and anaphylactoid reaction are classified according to the involvement of such antibodies as immuoglobulin $\mathrm{E}(\mathrm{IgE})$. But it is not easy to make a differential diagnosis of these two reactions based on the clinical symptoms. Instead, they can be classified according to skin test or biologic test. In association with this, it would be desirable to use such terms as anaphylactoid reaction in cases in which a diagnosis of immunological mechanisms was not made through an allergic test.

In regard to most cases of the allergy occurring during anesthesia, i.e., acute hypersensitivity reaction, grade- 2 responses (non-life-threatening skin reaction, hypotension accompanying tachycardia, coughing or the difficulty of mechanical respiration) or grade-3 responses (life-threatening cardiovascular collapse, bradycardia or tachycardia and severe bronchospasm) account for approximately $85 \%$ of total cases [4]

The current case is the one that is suspected to be grade- 2 or grade- 3 anaphylaxis occurring due to rocuronium. To make an accurate diagnosis of anaphylaxis or anaphylactoid reaction, in addition to clinical symptoms, the concentrations of tryptase or $\operatorname{IgE}$ which are present in the blood should be measured. After several weeks following the occurrence of allergic reactions, skin prick test or intradermal test should be performed [5]. Skin reactions occurring during the allergic responses due to drugs may be manifested as urticaria, edema, pruritis, thermal sensation and red spots.
On the other hand, Propofol may also cause the occurrence of anaphylaxis rarely. In the previous anaesthetic exposure, Propofol and rocuronium were not used. In this occasion, propofol, rocuronium was used along with Fentanyl. Based on these findings, in the current case, the possibility for anaphylaxis due to Propofol could not be ruled out.

Anaphylaxis occurring during anaesthesia occurs at a frequency which is approximately four times higher in men than women [5]. In regard to the age, it occurs most frequently in people in their forties.

Muscle relaxants are the most common drugs that cause the occurrence of anaphylaxis to a life-threatening extent during anaesthesia, accounting for approximately $50-70 \%$ of cases $[6,7]$.

Anaphylaxis occurring due to muscle relaxants occurred the most frequently in people aged ten years or younger. Besides, it also occurred the most frequently in women aged in their thirties. Clinical characteristics due to rocuronium are mainly classified into two types: cardiovascular collapse and bronchospasm [2]. Generally in cases in which bronchospasm occurred due to anaphylaxis, due to the actions of alpha-1 elevating the blood pressure and those of beta- 2 relaxing the bronchial smooth muscles, the immediate administration of epinephrine is important. Besides, in cases of cardiovascular collapse due to anaphylaxis, fluid supply is generally done and Epinephrine and Phenylephrine are administered. In cases which are refractory to these treatments, the administration of vasopressin could be of help [5].

Due to the increased permeability of capillary during the occurrence of anaphylaxis, the plasma volume is abruptly increased. A massive amount of fluid therapy might also be essential in this case. Owing to this, the oedema might occur in the lung or respiratory tract. This might be due to the increased permeability of pulmonary capillary vessels because of anaphylaxis. 
However in the case discussed here clinical or radiological evidence of pulmonary oedema did not occur. We assumed the possibility that pulmonary oedema occurred due to anaphylaxis or might have originated from the negative pressure of the respiratory tract

On the other hand, Rocuronium is an amino-steroid nondepolarizing muscle relaxant whose pharmacological characteristics are similar to those of Vecuronium. In cases of allergic reactions due to Rocuronium, there might be cross reactions with other types of steroid non-depolarizing muscle relaxants. In particular, aminosteroid muscle relaxants have a higher prevalence of the cross-reactions as compared with benzilisoquinoline-derived muscle relaxants [9]. These muscle relaxants commonly have tetra-positively charged ammonium ion $(\mathrm{NH} 4+)$ as an antigenic determinant. Due to this chemical structure, cross-reactions can occur. This should therefore be confirmed by further investigating the patient once stabilized [10].

The occurrence of Rocuronium induced anaphylaxis has been reported more frequently than that due to Vecuronium or Pancuronium and less frequently than that due to Succinylcholine [8]. There is a tendency that the use of Succinylcholine has recently been decreased and that of Rocuronium has been increased. Owing to this, the occurrence of rocuronium-induced anaphylaxis may be on the rise in western countries.

\section{Conclusion}

Muscle relaxants are the most commonly recognized cause for allergic reactions due to anaesthesia. Rocuronium is the number one cause for allergy among the muscle relaxants in western countries. Prompt identification and appropriate treatment according to established guidelines is important for immediate management of anaphylaxis. Follow up and further investigations for confirmation of anaphylactic reactions and identification of causative agent is important for prevention of further anaphylactic reactions during future anaesthesia.

\section{References}

ICU Management of a patient with Anaphylaxis

1. Mertes PM, Aimone-Gastin I, GueantRodriguez RM, Mouton- Faivre C, Audibert G, O'Brien J, et al. Hypersensitivity reactions to neuromuscular blocking agents. Curr Pharm Des 2008; 14: 2809-25.

2. Heier T, Guttormsen AB. Anaphylactic reactions during induction of anaesthesia using rocuronium for muscle relaxation: a report including 3 cases. Acta Anaesthesiol Scand 2000; 44:775-81.

3. Laxenaire MC, Mertes PM; Groupe d'Etudes des R e a c t i o n s Anaphylactoides Peranesthesiques. Anaphylaxis during anaesthesia: results of a two-year survey in France. Br J Anaesth 2001; 87: 549-58.

4. Laxenaire MC, Mertes PM; Groupe d'Etudes des Reactions Anaphylactoides Peranesthesiques. Anaphylaxis during anaesthesia: results of a two-year survey in France. Br J Anaesth 2001; 87: 549-58.

5. Rose M, Fisher M. Rocuronium: high risk for anaphylaxis? $\mathrm{Br} \mathrm{J}$ Anaesth 2001; 86: 678-82.

6. Mertes PM, Laxenaire MC. Allergy and anaphylaxis in anaesthesia. Minerva Anestesiol 2004; 70: 285-91.

7. Watkins J. Adverse reaction to neuromuscular blockers: frequency, investigation, and epidemiology. Acta Anaesthesiol Scand Suppl.1994; 102: 6-10.

8. Lee HM, Song SO. Anaphylaxis after injection of rocuronium: a case report. Korean J Anesthesiol 2006; 51: 101-4.

9. Laxenaire MC, Gastin I, MoneretVautrin DA, Widmer S, Gueant JL. Cross-reactivity of rocuronium with other neuromuscular blocking agents. Eur J Anaesthesiol Suppl 1995; 11: 55 64.

10.Matthey $\mathrm{P}$, Wang $\mathrm{P}$, Finegan BA, Donnelly M. Rocuronium anaphylaxis and multiple neuromuscular blocking drug sensitivities. Can J Anaesth 2000; 47:890-3. 